\title{
Moderate hypothermia at warmer temperatures is safe in elective proximal and total arch surgery: Results in 665 patients
}

Ourania Preventza, MD, ${ }^{\mathrm{a}, \mathrm{b}, \mathrm{c}}$ Joseph S. Coselli, MD, ${ }^{\mathrm{a}, \mathrm{b}, \mathrm{c}}$ Andrea Garcia, MD, ${ }^{\mathrm{b}}$ Sarang Kashyap, MD, ${ }^{\mathrm{a}}$ Shahab Akvan, MD, ${ }^{a}$ Katherine H. Simpson, MS, ${ }^{d}$ Matt D. Price, MS, ${ }^{b}$ Faisal G. Bakaeen, MD,,${ }^{\mathrm{a}, \mathrm{b}, \mathrm{c}, \mathrm{d}}$ Lorraine D. Cornwell, MD, ${ }^{\mathrm{b}, \mathrm{c}, \mathrm{d}}$ Shuab Omer, MD, ${ }^{\mathrm{b}, \mathrm{c}, \mathrm{d}}$ Kim I. de la Cruz, MD, ${ }^{\mathrm{b}, \mathrm{c}}$ Scott A. LeMaire, MD, ${ }^{\mathrm{a}, \mathrm{b}, \mathrm{c}}$ and Denton A. Cooley, MD ${ }^{\mathrm{a}, \mathrm{b}}$

\section{ABSTRACT}

Objective: To evaluate adverse outcomes after elective aortic arch surgery performed at higher or lower temperatures $\left(24.0^{\circ} \mathrm{C}-28.0^{\circ} \mathrm{C}\right.$ vs $\left.20.1^{\circ} \mathrm{C}-23.9^{\circ} \mathrm{C}\right)$ within the wide range of moderate hypothermia.

Methods: Over a 9-year period, a total of 665 patients underwent elective proximal $(n=479)$ or total $(n=186)$ arch replacement with moderate hypothermia and antegrade cerebral perfusion. Circulatory arrest was initiated at an actual temperature of $20.1^{\circ} \mathrm{C}$ to $23.9^{\circ} \mathrm{C}$ in the lower-temperature group $(\mathrm{n}=334 ; 223$ proximal, 111 total) and at $24.0^{\circ} \mathrm{C}$ to $28.0^{\circ} \mathrm{C}$ in the higher-temperature group ( $\mathrm{n}=331 ; 256$ proximal, 75 total). Composite adverse outcome was defined as operative mortality or persistent neurologic event or persistent hemodialysis at discharge. Multivariate logistic regression analysis was used to model adverse outcome. In addition to the actual temperature, a new, balanced variable, "predicted temperature," was analyzed to eliminate surgeon bias. We used this variable in a propensity score-matching analysis to validate the multivariate analysis results.

Results: A composite adverse outcome occurred in $7.2 \%$ of cases. Operative mortality was $5.1 \%$. The rate of postoperative persistent neurologic deficits was $2.4 \%$. No significant differences were found between the lower- and higher-predicted temperature groups within the moderate hypothermia range in the propensity score-matching analysis. The higher-actual temperature group had a lower rate of ventilator support at $>48$ hours $(P=.036)$ and less need for tracheostomy $(P=.023)$. Packed red blood cell transfusion and previous coronary artery bypass independently predicted composite adverse outcome $(P=.0053$ and .0002 , respectively), operative mortality $(P=.0051$ and .0041$)$, and postoperative stroke $(P=.045$ and .048$)$. Cardiopulmonary bypass time independently predicted composite outcome $(P=.0005)$, operative mortality $(P<.0001)$, ventilatory support for $>48$ hours $(P<.0001)$, and renal dysfunction $(P=.0005)$.

Conclusions: In elective proximal or total arch surgery, higher temperatures $\left(\geq 24.0^{\circ} \mathrm{C}-28.0^{\circ} \mathrm{C}\right)$ within the wide range of moderate hypothermia $\left(20.1^{\circ} \mathrm{C}-28^{\circ} \mathrm{C}\right)$ are safe and, compared with colder temperatures, not associated with significantly different rates of composite and adverse outcomes. (J Thorac Cardiovasc Surg 2017;153:1011-8)

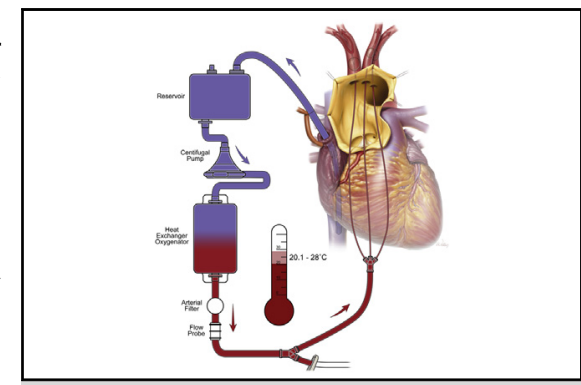

Higher temperatures in the moderate hypothermia range are safe for elective proximal and total arch surgery.

\section{Central Message}

Elective proximal and total arch surgery is safe at higher temperatures $\left(\geq 24.0^{\circ} \mathrm{C}-28.0^{\circ} \mathrm{C}\right)$ in the moderate hypothermia range $\left(20.1^{\circ} \mathrm{C}\right.$ $28^{\circ} \mathrm{C}$ ).

\section{Perspective}

Although centers of excellence still use cold temperatures for optimum brain protection, there is a clear trend toward increased use of antegrade cerebral perfusion, most notably with both moderate $\left(20.1^{\circ} \mathrm{C}-28^{\circ} \mathrm{C}\right)$ and mild hypothermia. Higher temperatures $\left(\geq 24.0^{\circ} \mathrm{C}\right.$ $28.0^{\circ} \mathrm{C}$ ) within the wide range of the moderate hypothermia $\left(20.1^{\circ} \mathrm{C}-28^{\circ} \mathrm{C}\right)$ are safe during elective arch surgery.

See Editorial Commentary page 1019.

\footnotetext{
From the ${ }^{\mathrm{a} D e p a r t m e n t}$ of Cardiovascular Surgery, Texas Heart Institute; ${ }^{\mathrm{b}}$ Division of Cardiothoracic Surgery, Michael E. DeBakey Department of Surgery, and ${ }^{\mathrm{C} C a r d i o-}$ vascular Research Institute, Baylor College of Medicine; and ${ }^{\mathrm{d}}$ Michael E. DeBakey Veterans Affairs Medical Center, Houston, Tex.

Received for publication April 15, 2016; revisions received Aug 30, 2016; accepted for publication Sept 9, 2016; available ahead of print Oct 22, 2016.
}

Address for reprints: Ourania Preventza, MD, BCM 390 One Baylor Plaza, Houston, TX 77030 (E-mail: opsmile01@aol.com). $0022-5223 / \$ 36.00$

Copyright (C) 2016 by The American Association for Thoracic Surgery http://dx.doi.org/10.1016/j.jtcvs.2016.09.044 


\section{Abbreviations and Acronyms}

$\mathrm{ACP}=$ antegrade cerebral perfusion

$\mathrm{CABG}=$ coronary artery bypass grafting

$\mathrm{CPB}=$ cardiopulmonary bypass

HCA = hypothermic circulatory arrest

$\mathrm{MOF}=$ multiple organ failure

Scanning this QR code will take you to a video for the article.

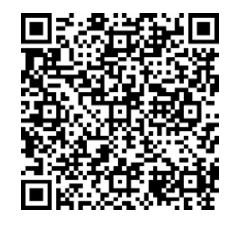

Various cerebral perfusion strategies, including antegrade cerebral perfusion (ACP), retrograde cerebral perfusion, and deep hypothermia alone, have been demonstrated to provide adequate brain protection during aortic arch operations. ${ }^{1-11}$ The debate regarding which strategy provides optimum brain protection is ongoing, but there has been a general shift toward favoring ACP and moderate degrees of hypothermia. ${ }^{1,7,9-11}$ According to Yan and colleagues' classification of hypothermia in aortic arch surgery, ${ }^{12}$ moderate hypothermia is defined as $20.1^{\circ} \mathrm{C}$ to $28^{\circ} \mathrm{C}$. The effects of higher or lower temperatures within this wide range on adverse outcome rates have not been well studied; specifically, data are lacking on the effect of temperature on outcomes of elective cases. In the present study of patients undergoing elective proximal and total arch surgery under moderate hypothermia $\left(20.1^{\circ} \mathrm{C}-28^{\circ} \mathrm{C}\right)$, we evaluated the effect of higher versus lower moderate hypothermic temperatures (ie, $\geq 24.0^{\circ} \mathrm{C}-28.0^{\circ} \mathrm{C}$ vs $\leq 20.1^{\circ} \mathrm{C}-23.9^{\circ} \mathrm{C}$ ) on adverse outcome.

\section{METHODS}

Over a 9-year period from March 2005 to December 2014, a total of 665 patients underwent elective proximal or total arch surgery at CHI St Luke's Health-Baylor St Luke's Medical Cente under a wide range of moderate hypothermic temperatures $\left(20.1^{\circ} \mathrm{C}-28^{\circ} \mathrm{C}\right.$ ) (Table 1). All patients received selective ACP for cerebral protection. Data were collected from a prospectively maintained database, and the project was approved by the Institutional Review Board at Baylor College of Medicine. Informed consent for data collection was obtained from all patients. Hospital records, admission and discharge summaries, operative diagrams, operative reports, and anesthesia and perfusion records were reviewed.

The patients were divided into 2 groups according to the actual nasopharyngeal temperature at the onset of circulatory arrest: $20.1^{\circ} \mathrm{C}$ to $23.9^{\circ} \mathrm{C}$ for the lower-temperature group $(\mathrm{n}=334)$ and $24.0^{\circ} \mathrm{C}$ to $28.0^{\circ} \mathrm{C}$ for the higher-temperature group $(\mathrm{n}=331)$. There were more patients with redo sternotomy and previous coronary artery bypass grafting $(\mathrm{CABG})$ in the lower-temperature group.

The predicted temperature was defined as a balancing score used to eliminate surgeon bias. This variable was the result of modeling actual temperatures and other preoperative and intraoperative variables (see Statistical Analysis for more details). There were 334 patients at the predicted lower temperatures $\left(\leq 20.1{ }^{\circ} \mathrm{C}-23.9^{\circ} \mathrm{C}\right)$ and 331 patients at the predicted higher temperatures $\left(\geq 24.0^{\circ} \mathrm{C}-28.0^{\circ} \mathrm{C}\right)$. The mean actual and predicted temperatures were $23.9^{\circ} \mathrm{C}$, and the median actual and predicted temperatures of all patients were $23.9^{\circ} \mathrm{C}$ (Figure 1). The cutoff for each group was based on a combination of our current practice experience, mean and median actual and predicted temperatures, and histogram findings. Almost equal numbers of patients were sorted into the 2 analysis groups according to this cutoff.

The ACP time was defined as the period during circulatory arrest in which the patient received ACP. The pure circulatory arrest time was the time without ACP or distal perfusion. The cases in which pure circulatory arrest was used in addition to ACP were usually redo cases in which cardiopulmonary bypass (CPB) had to be established before the sternum was opened and in which the right axillary artery was too small to allow cannulation. In these cases, the femoral artery was used for arterial inflow, and ACP was initiated after the chest was opened and the necessary dissection was performed. The CPB time was the period of CPB minus the pure circulatory arrest time and the ACP time. The myocardial ischemic time was the period during which the aorta was clamped or circulatory arrest occurred. Adverse outcome was defined as operative mortality, persistent neurologic event (stroke or spinal cord injury with persistent deficit at hospital discharge or at time of operative death), or persistent renal failure (marked by the need for hemodialysis at the time of hospital discharge or the time of operative death). Preoperative pulmonary disease, renal disease, and the postoperative outcomes have been previously defined. ${ }^{13}$ Operative mortality was defined as death before hospital discharge or within 30 days of operation, and postoperative stroke was defined as clinically or radiographically evident new brain injury after the procedure. Postoperative renal injury was defined as the need to start dialysis or a doubling of the patient's serum creatinine level compared with the baseline value. ${ }^{13}$

Our surgical technique for elective proximal and total arch surgery has been described previously. ${ }^{13,14}$ In the lower-actual temperature group, 81 patients received unilateral ACP and 253 received bilateral ACP. In the higher-actual temperature group, 84 patients received unilateral ACP and 247 received bilateral ACP. Intraoperative details for the 2 groups are provided in Table 2. Initially, we used bilateral ACP selectively for patients with longer circulatory arrest times ( $>20-30$ minutes) and those with short circulatory arrest times and a change of $>10 \%$ in the near-infrared spectroscopy measurement. Lately, bilateral ACP has been our default preference because it can be quickly and easily performed and does not add complexity to the operation. Elective proximal arch surgery was performed in 479 patients (223 in the lower-actual temperature group and 256 in the higher-actual temperature group), and total arch surgery was performed in 186 patients (111 in the lower-actual temperature group and 75 in the higher-actual temperature group) $(P=.0024)$ (Video 1).

\section{Statistical Analysis}

Univariate analysis of categorical data was performed using the $\chi^{2}$ test or Fisher exact test when necessary. For continuous variables, the Wilcoxon 2-sample test was used. Multivariate analysis consisted of both nominal logistic regression and standard least squares regression, both using stepwise selection. A total of 23 preoperative and 
TABLE 1. Preoperative characteristics and demographics of the total cohort (actual temperatures) and the propensity-matched cohort (predicted temperatures)

\begin{tabular}{|c|c|c|c|c|c|c|c|c|}
\hline \multirow[b]{2}{*}{ Characteristics } & \multicolumn{4}{|c|}{$\begin{array}{l}\text { Total cohort }(n=665) \\
\text { actual temperature }\end{array}$} & \multicolumn{4}{|c|}{$\begin{array}{c}\text { Propensity-matched cohort }(n=250) \text {, } \\
\text { predicted temperature }\end{array}$} \\
\hline & $\begin{array}{l}\text { Both groups } \\
(\mathrm{n}=665)\end{array}$ & $\begin{array}{c}\text { Lower- } \\
\text { temperature } \\
\text { group } \\
(\mathbf{n}=\mathbf{3 3 4}) \\
\end{array}$ & $\begin{array}{c}\text { Higher- } \\
\text { temperature } \\
\text { group } \\
(\mathbf{n}=\mathbf{3 3 1}) \\
\end{array}$ & $\begin{array}{c}P \\
\text { value }\end{array}$ & $\begin{array}{c}\text { Both } \\
\text { groups } \\
(\mathbf{n}=\mathbf{2 5 0}) \\
\end{array}$ & $\begin{array}{c}\text { Lower- } \\
\text { temperature } \\
\text { group } \\
(\mathbf{n}=\mathbf{1 2 5}) \\
\end{array}$ & $\begin{array}{c}\text { Higher- } \\
\text { temperature } \\
\text { group } \\
(\mathbf{n}=\mathbf{1 2 5}) \\
\end{array}$ & $\begin{array}{c}P \\
\text { value }\end{array}$ \\
\hline Age, y, median (IQR) & $62(53-71)$ & $63(54-71)$ & $61(52-71)$ & .36 & $63(54-72)$ & $63(53-72)$ & $64(56-72)$ & .50 \\
\hline Male sex, $\mathrm{n}(\%)$ & $475(71.4)$ & $230(68.9)$ & $245(74.0)$ & .14 & $168(67.2)$ & $83(66.4)$ & $85(68.0)$ & .79 \\
\hline Smoking, n (\%) & $289(43.5)$ & $150(44.9)$ & $139(42.0)$ & .45 & $118(47.2)$ & $58(46.4)$ & $60(48.0)$ & .80 \\
\hline Redo sternotomy, n (\%) & $229(34.4)$ & $136(40.7)$ & $93(28.1)$ & .0006 & $86(34.4)$ & $44(35.2)$ & $42(33.6)$ & .79 \\
\hline Hypertension, n (\%) & $533(80.2)$ & $263(78.7)$ & $270(81.6)$ & .36 & $197(78.8)$ & $100(80.0)$ & 97 (77.6) & .64 \\
\hline COPD, n $(\%)$ & $236(35.5)$ & $118(35.3)$ & $118(35.7)$ & .93 & $90(36.0)$ & $44(35.2)$ & $46(36.8)$ & .79 \\
\hline Preoperative cardiac disease, $\mathrm{n}(\%)^{*}$ & 257 (38.7) & $137(41.0)$ & $120(36.3)$ & .21 & $104(41.6)$ & $54(43.2)$ & $50(40.0)$ & .61 \\
\hline $\begin{array}{l}\text { Congestive heart failure } \\
\text { (NYHA III, IV), n (\%) }\end{array}$ & $156(23.5)$ & $88(26.4)$ & $68(20.5)$ & .077 & $62(24.8)$ & 37 (29.6) & $25(20.0)$ & .079 \\
\hline Renal disease, $\mathrm{n}(\%) \dagger$ & $71(10.7)$ & $35(10.5)$ & $36(10.9)$ & .87 & $29(11.6)$ & $17(13.6)$ & $12(9.6)$ & .32 \\
\hline History of stroke/TIA, n (\%) & $73(11.0)$ & $42(12.6)$ & $31(9.4)$ & .19 & $31(12.4)$ & $19(15.2)$ & $12(9.6)$ & .18 \\
\hline Diabetes mellitus, n (\%) & $58(8.7)$ & $35(10.5)$ & $23(7.0)$ & .11 & $24(9.6)$ & $11(8.8)$ & $13(10.4)$ & .67 \\
\hline Previous CABG surgery, $\mathrm{n}(\%)$ & $47(7.1)$ & $32(9.6)$ & $15(4.5)$ & .011 & $18(7.2)$ & $11(8.8)$ & $7(5.6)$ & .33 \\
\hline
\end{tabular}

$I Q R$, Interquartile range; $C O P D$, chronic obstructive pulmonary disease; NYHA, New York Heart Association; TIA, transient ischemic attack; CABG, coronary artery bypass grafting. *Preoperative cardiac disease was defined as preoperative myocardial infarction, coronary artery bypass grafting, percutaneous transluminal coronary angioplasty, coronary artery disease, or pacemaker placement. $\dagger$ Serum creatinine level $\geq 1.5 \mathrm{mg} / \mathrm{dL}$ or history of hemodialysis.

intraoperative variables were considered for inclusion in the models: year of surgery, type of surgery (total arch replacement vs proximal arch surgery), actual temperature at initiation of circulatory arrest, age, sex, smoking, redo sternotomy, hypertension, chronic obstructive pulmonary disease, preoperative coronary event, congestive heart failure, renal dysfunction, stroke or transient ischemic attack, diabetes mellitus, previous CABG surgery, concomitant CABG surgery, concomitant aortic root replacement, intraoperative packed red blood cell (PRBC) transfusion, unilateral versus bilateral ACP, CPB time, myocardial ischemia time, circulatory arrest time, and ACP time.
Because some outcomes were infrequent (eg, 34 deaths), we needed to reduce this set of variables to avoid overfitting the models; thus, we chose 14 of these 23 predictor variables to use in the models because they differed significantly between the 2 groups in univariate analysis or because they were reported as independent predictors in other studies.

Logistic regression models were run for the following binary outcomes: composite adverse event, operative mortality, postoperative stroke, postoperative pulmonary event, and postoperative renal event. A stepwise selection option was used. For sensitivity analysis, the models were run
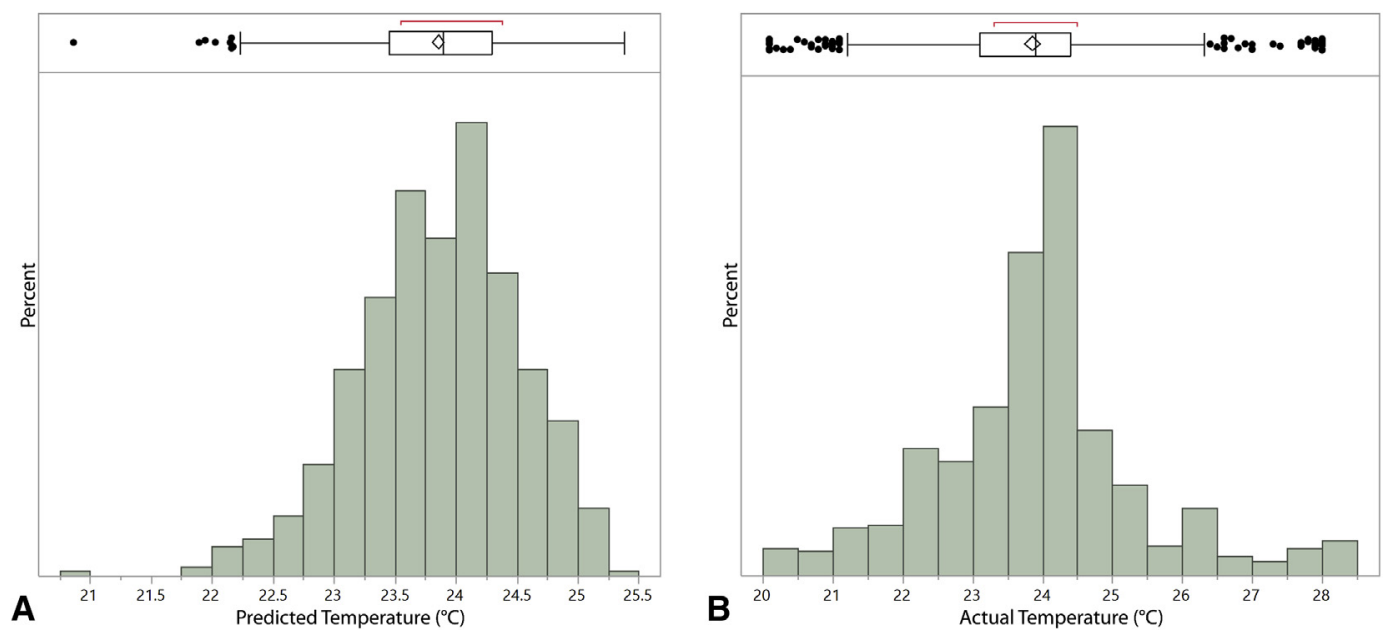

FIGURE 1. Histograms showing the distributions of the predicted temperatures (A) and actual temperatures (B) at which circulatory arrest was initiated. The mean actual and predicted temperatures were $23.9^{\circ} \mathrm{C}$, and the median actual and predicted temperatures of all the patients were $23.9^{\circ} \mathrm{C}$. 
TABLE 2. Intraoperative details for the total cohort (actual temperatures) and the propensity-matched cohort (predicted temperatures)

\begin{tabular}{|c|c|c|c|c|c|c|c|c|}
\hline \multirow[b]{2}{*}{ Intraoperative details } & \multicolumn{4}{|c|}{$\begin{array}{l}\text { Total cohort }(n=665) \\
\text { actual temperature }\end{array}$} & \multicolumn{4}{|c|}{$\begin{array}{c}\text { Propensity-matched cohort }(n=250), \\
\text { predicted temperature }\end{array}$} \\
\hline & $\begin{array}{l}\text { Both groups } \\
\quad(n=665)\end{array}$ & $\begin{array}{c}\text { Lower- } \\
\text { temperature } \\
\text { group } \\
(\mathbf{n}=\mathbf{3 3 4})\end{array}$ & $\begin{array}{c}\text { Higher- } \\
\text { temperature } \\
\text { group } \\
(\mathbf{n}=\mathbf{3 3 1})\end{array}$ & $\begin{array}{c}P \\
\text { value }\end{array}$ & $\begin{array}{c}\text { Both } \\
\text { groups } \\
(\mathbf{n}=\mathbf{2 5 0})\end{array}$ & $\begin{array}{c}\text { Lower- } \\
\text { temperature } \\
\text { group } \\
(\mathbf{n}=\mathbf{1 2 5})\end{array}$ & $\begin{array}{c}\text { Higher- } \\
\text { temperature } \\
\text { group } \\
(\mathbf{n}=\mathbf{1 2 5})\end{array}$ & $\begin{array}{c}P \\
\text { value }\end{array}$ \\
\hline Procedure, $\mathrm{n}(\%)$ & & & & .0024 & & & & .68 \\
\hline Total arch replacement & $186(28.0)$ & $111(33.2)$ & $75(22.7)$ & & $73(29.2)$ & $38(30.4)$ & $35(28.0)$ & \\
\hline Proximal arch surgery & $479(72.0)$ & $223(66.8)$ & $256(77.3)$ & & $177(70.8)$ & $87(69.6)$ & $90(72.0)$ & \\
\hline \multicolumn{9}{|l|}{ Concomitant procedures, $\mathrm{n}(\%)$} \\
\hline Aortic root replacement & $217(32.6)$ & $99(29.6)$ & $118(35.7)$ & .10 & $62(24.8)$ & $32(25.6)$ & $30(24.0)$ & .77 \\
\hline CABG & $106(15.9)$ & $46(13.8)$ & $60(18.1)$ & .13 & $41(16.4)$ & $21(16.8)$ & $20(16.0)$ & .86 \\
\hline Maze/patent foramen ovale repair & $32(4.8)$ & $14(4.2)$ & $18(5.4)$ & .45 & $12(4.8)$ & $5(4.0)$ & $7(5.6)$ & .55 \\
\hline Mitral valve repair/replacement & $14(2.1)$ & $9(2.7)$ & $5(1.5)$ & .29 & $6(2.4)$ & $3(2.4)$ & $3(2.4)$ & 1.00 \\
\hline Tricuspid valve repair/replacement & $1(0.2)$ & $1(0.3)$ & $0(0.0)$ & 1.00 & - & - & - & - \\
\hline Intraoperative PRBCs, n (\%) & $382(57.6)$ & $196(59.0)$ & $186(56.2)$ & .46 & $164(65.6)$ & $84(67.2)$ & $80(64.0)$ & .59 \\
\hline $\mathrm{ACP}, \mathrm{n}(\%)$ & & & & .74 & & & & .55 \\
\hline Unilateral & $165(24.8)$ & $81(24.3)$ & $84(25.4)$ & & $60(24.0)$ & $32(25.6)$ & $28(22.4)$ & \\
\hline Bilateral & $500(75.2)$ & $253(75.8)$ & 247 (74.6) & & $190(76.0)$ & $93(74.4)$ & $97(77.6)$ & \\
\hline \multicolumn{9}{|l|}{ Operative time (min), median (IQR) } \\
\hline $\mathrm{CPB}$ & $131(104-172)$ & $135.5(104-178)$ & $130(104-164)$ & .20 & $125(99-164)$ & $125(103-164)$ & $125(98-156)$ & .70 \\
\hline Myocardial ischemia & $90(66-118)$ & $89(66-120)$ & $91(68-117)$ & .63 & $84(65-111)$ & $87(66-110)$ & $82(64-112)$ & .78 \\
\hline Circulatory arrest & $24(17-48)$ & $28(18-56)$ & $22(16-35)$ & $<.0001$ & $24(18-44)$ & $26(18-44)$ & $23(18-46)$ & .71 \\
\hline $\mathrm{ACP}$ & $23(17-47)$ & $27(18-55)$ & $22(16-35)$ & $<.0001$ & $23.5(18-44)$ & $26(18-43)$ & $22(17-46)$ & .57 \\
\hline
\end{tabular}

$C A B G$, Coronary artery bypass grafting; $P R B C s$, packed red blood cells; $A C P$, antegrade cerebral perfusion; $I Q R$, interquartile range; $C P B$, cardiopulmonary bypass.

again with a relaxed variable stay $P$ value of .10 . In addition, the predicted temperature variable was used as a balancing score (ie, generalized propensity score). The predicted temperature was the result of modeling the actual temperature as the dependent variable and the following factors as independent variables: year, age, male sex, smoker, redo sternotomy, hypertension, chronic obstructive pulmonary disease, preoperative coronary event, congestive heart failure, renal failure, preoperative neurologic deficits, diabetes, previous $\mathrm{CABG}$, total arch replacement,

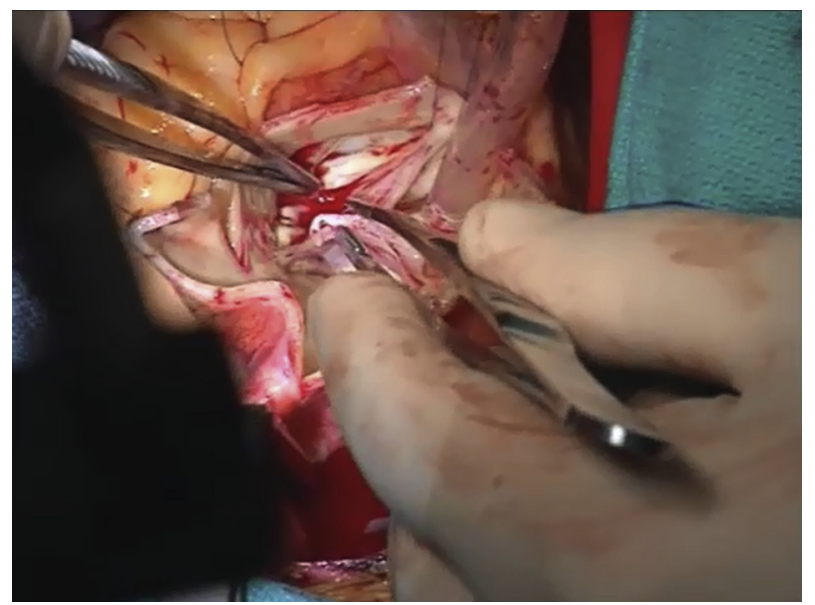

VIDEO 1. Hemiarch replacement and aortic valve-sparing procedure under moderate hypothermia. Video available at: http://www.jtcvsonline. org/article/S0022-5223(16)31162-X/addons. concomitant aortic root replacement, concomitant CABG, concomitant maze procedure, concomitant mitral valve replacement, intraoperative $\mathrm{PRBC}$ transfusion, bilateral $\mathrm{ACP}, \mathrm{CPB}$ time, cardiac ischemia time, and ACP time. A propensity score was created for each patient using a multivariate stepwise logistic regression model $(\mathrm{C}$-index $=0.90$; Hosmer-Lemeshow goodness-of-fit $P$ value $=.20$ ). Propensity matching was then performed for the patients using a 1-to-1 greedy match algorithm without replacement, which resulted in 125 matched pairs. Differences in the distributions of preoperative, operative, and postoperative characteristics between these lower- and higher-predicted temperature groups were tested using the $\chi^{2}$ test (or the Fisher exact test when necessary) for the categorical variables and the nonparametric Wilcoxon 2-sample test for the continuous variables. This process allowed us to validate the multivariate model results for the actual temperatures, which were affected by physician bias.

A histogram was created from the actual and the predicted temperatures (Figure 1). All statistical analyses were conducted with SAS version 9.1 (SAS Institute, Cary, NC) and JMP version 12.2 (SAS Institute). Statistical significance was determined by $P<.05$.

\section{RESULTS \\ Operative Mortality}

The overall operative mortality in our series was $5.1 \%$ $(\mathrm{n}=34)$. Sixteen patients $(4.8 \%)$ died in the lower-actual temperature group, compared with 18 patients $(5.4 \%)$ in the higher-actual temperature group $(P=.70)$. In the lower-actual temperature group, 2 patients sustained fatal stroke, 4 patients died of multiple organ failure (MOF), and 5 patients died of heart failure and an inability to wean from extracorporeal membrane oxygenation. One 
TABLE 3. Short-term complications in the total cohort (actual temperatures) and the propensity-matched cohort (predicted temperatures)

\begin{tabular}{|c|c|c|c|c|c|c|c|c|}
\hline \multirow[b]{2}{*}{ Complication } & \multicolumn{4}{|c|}{$\begin{array}{l}\text { Total cohort }(n=665) \\
\text { actual temperature }\end{array}$} & \multicolumn{4}{|c|}{$\begin{array}{c}\text { Propensity-matched cohort }(n=250), \\
\text { predicted temperature }\end{array}$} \\
\hline & $\begin{array}{c}\text { Both groups } \\
(\mathbf{n}=665)\end{array}$ & $\begin{array}{c}\text { Lower- } \\
\text { temperature } \\
\text { group } \\
(\mathbf{n}=\mathbf{3 3 4})\end{array}$ & $\begin{array}{c}\text { Higher- } \\
\text { temperature } \\
\text { group } \\
(\mathbf{n}=\mathbf{3 3 1})\end{array}$ & $\begin{array}{c}P \\
\text { value }\end{array}$ & $\begin{array}{l}\text { Both groups } \\
\quad(\mathbf{n}=\mathbf{2 5 0})\end{array}$ & $\begin{array}{c}\text { Lower- } \\
\text { temperature } \\
\text { group } \\
(\mathbf{n}=\mathbf{1 2 5})\end{array}$ & $\begin{array}{c}\text { Higher- } \\
\text { temperature } \\
\text { group } \\
(\mathbf{n}=\mathbf{1 2 5})\end{array}$ & $\begin{array}{c}P \\
\text { value }\end{array}$ \\
\hline Composite adverse outcome, $\mathrm{n}(\%)^{*}$ & $48(7.2)$ & $23(6.9)$ & $25(7.6)$ & .74 & $20(8.0)$ & $13(10.4)$ & $7(5.6)$ & .16 \\
\hline Operative mortality, n (\%) & $34(5.1)$ & $16(4.8)$ & $18(5.4)$ & .70 & $15(6.0)$ & $9(7.2)$ & $6(4.8)$ & .42 \\
\hline Total neurologic events, n (\%) & $26(3.9)$ & $14(4.2)$ & $12(3.6)$ & .71 & $10(4.0)$ & $6(4.8)$ & $4(3.2)$ & .52 \\
\hline Total persistent neurologic events, $\mathrm{n}(\%)$ & $16(2.4)$ & $7(2.1)$ & $9(2.7)$ & .60 & $7(2.8)$ & $5(4.0)$ & $2(1.6)$ & .45 \\
\hline Stroke, n $(\%)$ & $23(3.5)$ & $12(3.6)$ & $11(3.3)$ & .85 & $7(2.8)$ & $5(4.0)$ & $2(1.6)$ & .45 \\
\hline Persistent stroke, n (\%) & $14(2.1)$ & $5(1.5)$ & $9(2.7)$ & .27 & $5(2.0)$ & $4(3.2)$ & $1(0.8)$ & .37 \\
\hline All renal dysfunction, $\mathrm{n}(\%) \dagger$ & $83(12.5)$ & $40(12.0)$ & $43(13.0)$ & .69 & $30(12.0)$ & $13(10.4)$ & $17(13.6)$ & .44 \\
\hline Persistent renal failure, $\mathrm{n}(\%) \ddagger$ & $3(0.5)$ & $3(0.9)$ & $0(0.0)$ & .25 & $1(0.4)$ & $1(0.8)$ & $0(0.0)$ & 1.00 \\
\hline Ventilator support $>48 \mathrm{~h}, \mathrm{n}(\%)$ & $164(24.7)$ & $94(28.1)$ & $70(21.2)$ & .036 & $61(24.4)$ & $32(25.6)$ & $29(23.2)$ & .66 \\
\hline Tracheostomy, n (\%) & $59(8.9)$ & $38(11.4)$ & $21(6.4)$ & .023 & $23(9.2)$ & $15(12.0)$ & $8(6.4)$ & .13 \\
\hline Reoperation for bleeding, $\mathrm{n}(\%)$ & $37(5.8)$ & $15(4.7)$ & $22(7.0)$ & .22 & $13(5.5)$ & $6(5.3)$ & $7(5.6)$ & .92 \\
\hline Postoperative deep venous thrombosis, $\mathrm{n}(\%)$ & $24(3.6)$ & $15(4.5)$ & $9(2.8)$ & .24 & $11(4.4)$ & $8(6.4)$ & $3(2.4)$ & .13 \\
\hline Postoperative pericardial window, n (\%) & $16(2.4)$ & $8(2.4)$ & $8(2.4)$ & .97 & $4(1.6)$ & $3(2.4)$ & $1(0.8)$ & .62 \\
\hline Postoperative myocardial infarction, n (\%) & $8(1.2)$ & $4(1.2)$ & $4(1.2)$ & 1.00 & $3(1.2)$ & $3(2.4)$ & $0(0.0)$ & .25 \\
\hline Postoperative pulmonary embolism, n (\%) & $3(0.5)$ & $1(0.3)$ & $2(0.6)$ & .62 & - & - & - & - \\
\hline
\end{tabular}

*Composite adverse outcome was defined as operative mortality, persistent neurologic event, or persistent hemodialysis at discharge. $\nmid$ Postoperative renal dysfunction with or without temporary or persistent hemodialysis. $\ddagger$ Hemodialysis dependence at discharge.

patient experienced primary cardiac arrest and died of anoxic brain injury. One patient developed toxic megacolon, underwent total colectomy, and died of sepsis and MOF. One patient underwent transvenous pacemaker placement by the cardiology service for complete heart block and shortly thereafter could not be resuscitated from a sudden cardiac arrest. One patient received emergency extracorporeal membrane oxygenation support after cardiac arrest and could not be weaned. One patient had an uneventful recovery after aortic root and proximal arch replacement, was discharged on postoperative day 11 , but died a week later while at home (Table 3).

In the higher-actual temperature group, 7 patients died of heart failure, 5 died of MOF, and 2 died of stroke. Two patients died after discharge but within 30 days of surgery while recovering at home. Two patients died of anoxic brain injury after cardiopulmonary resuscitation (Table 3).

Whether circulatory arrest was initiated at a higher or lower actual temperature was unrelated to mortality in the multivariate analysis. The propensity score-matching analysis based on the predicted temperature validated this finding. Independent factors associated with operative mortality in the full cohort were increasing CPB time $(P<.0001)$, intraoperative PRBC transfusion $(P=.0053)$, and previous CABG surgery $(P=.0041)$ (Table 4).

\section{Neurologic Events}

Five patients in the lower-actual temperature group had persistent stroke $(1.5 \%), 2$ of whom died. Two patients had spinal cord ischemia ( 1 with persistent paralysis after frozen elephant trunk and 1 with persistent paraparesis after total arch and elephant trunk creation).

Nine patients in the higher-actual temperature group $(2.7 \%)$ had persistent stroke, 2 of whom died. No spinal cord ischemia was observed in this group. Rates of all neurologic events and persistent neurologic events were not significantly different between the 2 groups, in both the entire cohort $(\mathrm{n}=665 ; P=.71$ and .60 , respectively) and the propensity-matched cohort $(\mathrm{n}=250 ; P=.52$ and .45 , respectively) (Table 3). Likewise, being in the actual-temperature group was not an independent predictor for stroke or persistent neurologic events in either the full cohort or the propensity-matched patients. Intraoperative PRBC transfusion and previous CABG were independently associated with postoperative stroke $(P=.046$ and .048 , respectively) (Table 4).

\section{Composite Adverse Outcome}

Twenty-three patients $(6.9 \%)$ in the lower-actual temperature group and 25 patients $(7.6 \%)$ in the higheractual temperature group had persistent adverse outcomes $(P=.74)$ (Table 3). Multivariate analysis showed identified the following independent factors for adverse outcome: 
TABLE 4. Odds ratios obtained by multivariate regression analysis

\begin{tabular}{|c|c|c|c|c|c|}
\hline Outcome variable & $\begin{array}{c}P \\
\text { value }\end{array}$ & OR & $95 \% \mathrm{CI}$ & $\begin{array}{c}\text { C- } \\
\text { index }\end{array}$ & $\begin{array}{c}\text { Hosmer- } \\
\text { Lemeshov } \\
P \text { value }\end{array}$ \\
\hline $\begin{array}{c}\text { Composite adverse } \\
\text { outcome }\end{array}$ & & & & 0.79 & .032 \\
\hline Previous CABG & .0002 & 4.38 & $2.02-9.51$ & & \\
\hline ACP time (per min) & .019 & 1.01 & $1.00-1.02$ & & \\
\hline Intraoperative PRBCs & .0053 & 4.02 & $1.51-10.69$ & & \\
\hline CPB time (per min) & .0005 & 1.01 & $1.00-1.01$ & & \\
\hline Operative variables & & & & 0.83 & .59 \\
\hline Previous CABG & .0041 & 3.77 & $1.52-9.31$ & & \\
\hline Intraoperative PRBCs & .0051 & 8.07 & $1.87-34.80$ & & \\
\hline CPB time (per min) & $<.0001$ & 1.01 & $1.01-1.02$ & & \\
\hline Postoperative stroke & & & & 0.67 & .92 \\
\hline Previous CABG & .048 & 2.91 & $1.01-8.42$ & & \\
\hline Intraoperative PRBCs & .045 & 3.12 & $1.03-9.48$ & & \\
\hline Ventilator support $>48 \mathrm{~h}$ & & & & 0.73 & 61 \\
\hline ACP time (per min) & $<.0001$ & 1.02 & $1.01-1.02$ & & \\
\hline Intraoperative PRBCs & .0016 & 2.02 & $1.31-3.12$ & & \\
\hline CPB time (per min) & $<.0001$ & 1.009 & $1.005-1.012$ & & \\
\hline All renal dysfunction* & & & & 0.72 & 1.00 \\
\hline ACP time (per min) & .0025 & 1.01 & $1.00-1.02$ & & \\
\hline Intraoperative PRBCs & .0041 & 2.35 & $1.31-4.21$ & & \\
\hline CPB time (per min) & .0005 & 1.01 & $1.00-1.01$ & & \\
\hline
\end{tabular}

$\overline{O R}$, Odds ratio; $C I$, confidence interval; $C A B G$, coronary artery bypass grafting; $A C P$, antegrade cerebral perfusion; $P R B C s$, packed red blood cells; $C P B$, cardiopulmonary bypass. *Postoperative renal dysfunction with or without temporary or persistent hemodialysis.

previous CABG $(P=.0002)$, intraoperative PRBC transfusion $(P=.0055)$, increasing $\mathrm{CPB}$ time $(P=.0005)$, and increasing ACP time $(P=.019)$ (Table 4). Propensity score-matching analysis of the predicted temperatures showed no difference between the 2 groups. Other short-term complications are presented in Table 3.

\section{DISCUSSION}

In 1975, Griepp and colleagues ${ }^{15}$ reported the first series of aortic arch operations in which profound hypothermia $\left(<15^{\circ} \mathrm{C}\right)$ was used to slow cerebral metabolism, allowing for safe induction of brief circulatory arrest. McCullough and colleagues ${ }^{16}$ used cerebral oxygen consumption data to estimate safe intervals of hypothermic circulatory arrest (HCA) at various temperatures and reported the following safe intervals: 9 minutes at $30^{\circ} \mathrm{C}, 14$ minutes at $25^{\circ} \mathrm{C}, 21$ minutes at $20^{\circ} \mathrm{C}, 31$ minutes at $15^{\circ} \mathrm{C}$, and 45 minutes at $10^{\circ} \mathrm{C}$. Since then, other studies ${ }^{17-19}$ have suggested that the combination of HCA and adjuncts, such as cerebral perfusion, offers superior results by maintaining the nervous system's metabolic supply. There has been a clear trend toward an increased use of ACP, most notably with both moderate and mild hypothermia, ${ }^{9-11,20,21}$ even though a meta-analysis of 5060 patients showed that retrograde cerebral perfusion and ACP provided similar cerebral protection when combined with deep hypothermia circulatory arrest, ${ }^{22}$ and experts at other centers of excellence have maintained that cold temperatures are preferable. ${ }^{2}$

Supporters of using warmer temperatures suggest that lower temperature decreases postoperative bleeding and operative time, but there is no strong scientific evidence to support this claim. In our study, warmer temperatures $\left(\geq 24.0^{\circ} \mathrm{C}-28.0^{\circ} \mathrm{C}\right)$ were associated with shorter CPB time and fewer intraoperative PRBC transfusions, but not significantly so $(P=.20$ and .46 , respectively). Total circulatory arrest and ACP times were longer in the lower-actual temperature group compared with the higher-actual temperature group $(P<.0001$ for both $)$, but this was expected because the lower-actual temperature group had a higher percentage of total arch repairs versus hemiarch repairs.

Disturbances of the coagulation system have been reported with profound hypothermia, ${ }^{23}$ and lower temperatures have been associated with reexploration for bleeding. Milewski and colleagues ${ }^{4}$ found that the use of profound $\mathrm{HCA}\left(<20^{\circ} \mathrm{C}\right)$ or deep HCA $\left(21^{\circ} \mathrm{C}-26^{\circ} \mathrm{C}\right)$ did not affect postoperative bleeding rates after open aortic arch surgery. We found no association between actual or predicted temperature and the incidence of postoperative bleeding; others have arrived at the same conclusion.

The frequency of intraoperative PRBC transfusion was slightly higher in the lower-actual temperature and lower-predicted temperature groups $\left(\leq 20.1^{\circ} \mathrm{C}-23.9^{\circ} \mathrm{C}\right)$. In the multivariate analysis, intraoperative PRBC transfusion was associated with adverse outcomes, operative mortality, stroke, ventilatory support for $>48$ hours, and postoperative renal dysfunction.

The operative mortality and persistent stroke rates in our higher-actual temperature group $\left(\geq 24.0^{\circ} \mathrm{C}-28.0^{\circ} \mathrm{C}\right)$ were similar to those in the elective subgroup described by Leshnower and colleagues, ${ }^{24}$ who reviewed the outcomes of 412 patients who underwent proximal or total arch surgery (including emergent cases). All patients received unilateral ACP, and the core temperature at the initiation of circulatory arrest was $25.7^{\circ} \mathrm{C} \pm 2.8^{\circ} \mathrm{C}$. In their series, increasing $\mathrm{CPB}$ time was an independent predictor of mortality. Likewise, in our series, increasing CPB time was associated with postoperative morbidity, operative mortality, and the composite adverse outcome on multivariate analysis (Table 4).

Leshnower and colleagues ${ }^{24}$ did not identify temperature as an independent risk factor for mortality, stroke, renal failure requiring dialysis, exploration for bleeding, or 
prolonged mechanical ventilation. Similarly, in our series, actual temperature within the moderate hypothermia range $\left(20.1^{\circ} \mathrm{C}-28^{\circ} \mathrm{C}\right)$ was not associated with adverse outcomes, operative mortality, or stroke by multivariate analysis, and the same results were found for the predicted temperature in the propensity-matching analysis; however, the lower-actual temperature group had higher rates of prolonged ventilatory support ( $>48$ hours; $P=.036)$ and respiratory failure necessitating tracheostomy $(P=.023)$ in the univariate analysis only. Among the patients who were propensity-matched according to their predicted temperature, higher rates of stroke and total neurologic events were seen in the lower-predicted temperature group, although the differences were not statistically significant.

The effect of higher temperature on visceral organ and spinal cord perfusion after aortic arch surgery may be of concern. No paralysis or paraparesis was seen in our higher-actual temperature group. Two cases of spinal cord ischemia occurred in our lower-actual temperature group $\left(\leq 20.1^{\circ} \mathrm{C}-23.9^{\circ} \mathrm{C}\right)$, likely associated with creation of an elephant trunk ( 1 of the 2 patients had frozen elephant trunk). Other groups have reported series without spinal cord ischemic events despite using higher temperatures ${ }^{21,24}$; however, prolonged circulatory arrest time at high temperatures must be applied with caution, given that the limits of depriving the lower body of blood flow are not strictly defined. No postoperative mesenteric ischemia or isolated liver failure was noted in our series. These results are supported by findings of previous studies. $^{25}$

Our persistent rate of renal failure (defined as the requirement for hemodialysis at discharge) was lower than our rate of postoperative temporary acute renal injury, but there was no significant difference between the 2 groups in the rates of either complication. These findings agree with those reported by Pacini and colleagues $^{26}$ in a series of 304 patients undergoing arch surgery. In their series, 25 patients $(8.2 \%)$ had isolated postoperative renal dysfunction, and 41 patients $(13.5 \%)$ had associated renal and liver dysfunction. Dialysis was temporary in 19 patients $(6.2 \%)$ and persistent in only 2 patients. Isolated liver dysfunction occurred in 69 patients $(22.7 \%)$, and only 3 patients $(1.0 \%)$ had major gastrointestinal complications. Importantly, when the patients were divided into 2 groups according to hypothermic temperature of $\leq 25^{\circ} \mathrm{C}(\mathrm{n}=194)$ or $>25^{\circ} \mathrm{C}(\mathrm{n}=110)$, the rates of each of these outcomes did not differ significantly between the groups. Prolonged CPB time was the sole significant independent risk factor for renal dysfunction, with or without liver dysfunction. This result is in agreement with our multivariate analysis findings (Table 4).
The main limitation of the present study is its retrospective design, which potentially incurs the biases associated with all such studies. We did not evaluate noncritical liver dysfunction, which would have manifested as enzyme-level elevation, and we did not analyze the long-term effects of higher temperatures on neurocognitive function. Moreover, our study is underpowered; having $80 \%$ power to detect a difference of this magnitude at an $\alpha$ value of 0.05 would require a much higher number of patients (in the range of 1500 patients per group).

In conclusion, within the wide range of temperatures $\left(20.1^{\circ} \mathrm{C}-28.0^{\circ} \mathrm{C}\right)$ used in moderate hypothermia, higher temperatures $\left(\geq 24.0^{\circ} \mathrm{C}-28.0^{\circ} \mathrm{C}\right)$ are safe and do not appear to be associated with adverse outcome when compared with lower temperatures. Given the shift toward the wider use of moderate hypothermia, these findings have practical relevance to the performance of aortic arch surgery.

\section{Conflict of Interest Statement}

Dr Coselli serves as PI for ongoing clinical trials with Edwards LifeSciences, Medtronic, Inc, and W.L. Gore \& Associates. He also consults with Vascutek Terumo, Medtronic, Inc, and W. L. Gore \& Associates. Dr Preventza is also a consultant for Medtronic, Inc and has had travel expenses paid by W.L. Gore \& Associates, Inc, and Cook Medical. Dr LeMaire serves as PI for clinical studies with Vascutek Terumo and Baxter Healthcare, and he has served as an advisory board member for Baxter Healthcare. All other authors have nothing to disclose with regard to commercial support.

The authors thank Kelly Tucker, MS, Department of Scientific Publications, Texas Heart Institute, for editorial support.

\section{References}

1. Bachet J. What is the best method for brain protection in surgery of the aortic arch? Selective antegrade cerebral perfusion. Cardiol Clin. 2010;28: 389-401.

2. Chau KH, Friedman T, Tranquilli M, Elefteriades JA. Deep hypothermic circulatory arrest effectively preserves neurocognitive function. Ann Thorac Surg. 2013;96:1553-9.

3. Ganapathi AM, Hanna JM, Schechter MA, Englum BR, Castleberry AW, Gaca JG, et al. Antegrade versus retrograde cerebral perfusion for hemiarch replacement with deep hypothermic circulatory arrest: does it matter? A propensity-matched analysis. J Thorac Cardiovasc Surg. 2014;148: 2896-902

4. Milewski RK, Pacini D, Moser GW, Moeller P, Cowie D, Szeto WY, et al Retrograde and antegrade cerebral perfusion: results in short elective arch reconstructive times. Ann Thorac Surg. 2010;89:1448-57.

5. Misfeld M, Leontyev S, Borger MA, Gindensperger O, Lehmann S, Legare JF et al. What is the best strategy for brain protection in patients undergoing aortic arch surgery? A single center experience of 636 patients. Ann Thorac Surg. 2012 93:1502-8. 
6. Okita Y, Miyata H, Motomura N, Takamoto S. A study of brain protection during total arch replacement comparing antegrade cerebral perfusion versus hypothermic circulatory arrest, with or without retrograde cerebral perfusion: analysis based on the Japan Adult Cardiovascular Surgery Database. J Thorac Cardiovasc Surg. 2015;149(2 Suppl):S65-73.

7. Spielvogel D, Kai M, Tang GH, Malekan R, Lansman SL. Selective cerebral perfusion: a review of the evidence. J Thorac Cardiovasc Surg. 2013;145(3 Suppl):S59-62.

8. Svensson LG, Blackstone EH, Apperson-Hansen C, Ruggieri PM, Ainkaran P, Naugle RI, et al. Implications from neurologic assessment of brain protection for total arch replacement from a randomized trial. J Thorac Cardiovasc Surg. 2015;150:1140-7.e11.

9. Tian DH, Wan B, Bannon PG, Misfeld M, LeMaire SA, Kazui T, et al. A meta-analysis of deep hypothermic circulatory arrest alone versus with adjunctive selective antegrade cerebral perfusion. Ann Cardiothorac Surg. 2013;2:261-70.

10. Tsai JY, Pan W, LeMaire SA, Pisklak P, Lee VV, Bracey AW, et al. Moderate hypothermia during aortic arch surgery is associated with reduced risk of early mortality. J Thorac Cardiovasc Surg. 2013;146:662-7.

11. Zierer A, El-Sayed Ahmad A, Papadopoulos N, Moritz A, Diegeler A, Urbanski PP. Selective antegrade cerebral perfusion and mild $\left(28^{\circ} \mathrm{C}-30^{\circ} \mathrm{C}\right)$ systemic hypothermic circulatory arrest for aortic arch replacement: results from 1002 patients. J Thorac Cardiovasc Surg. 2012;144:1042-9.

12. Yan TD, Bannon PG, Bavaria J, Coselli JS, Elefteriades JA, Griepp RB, et al. Consensus on hypothermia in aortic arch surgery. Ann Cardiothorac Surg. 2013;2:163-8.

13. Preventza O, Garcia A, Tuluca A, Henry M, Cooley DA, Simpson K, et al Innominate artery cannulation for proximal aortic surgery: outcomes and neurological events in 263 patients. Eur J Cardiothorac Surg. 2015;48: 937-42.

14. Preventza O, Garcia A, Cooley DA, Haywood-Watson RJ, Simpson K, Bakaeen FG, et al. Total aortic arch replacement: a comparative study of zone 0 hybrid arch exclusion versus traditional open repair. J Thorac Cardiovasc Surg. 2015;150:1591-8; discussion 1598-600.

15. Griepp RB, Stinson EB, Hollingsworth JF, Buehler D. Prosthetic replacement of the aortic arch. J Thorac Cardiovasc Surg. 1975;70:1051-63.

16. McCullough JN, Zhang N, Reich DL, Juvonen TS, Klein JJ, Spielvogel D, et al. Cerebral metabolic suppression during hypothermic circulatory arrest in humans. Ann Thorac Surg. 1999;67:1895-9; discussion 1919-21.
17. Guilmet D, Roux PM, Bachet J, Goudot B, Tawil N, Diaz F. A new technic of cerebral protection. Surgery of the aortic arch. Presse Med. 1986;15:1096-8 (in French).

18. Kazui T, Washiyama N, Muhammad BA, Terada H, Yamashita K. Takinami M. Improved results of atherosclerotic arch aneurysm operations with a refined technique. J Thorac Cardiovasc Surg. 2001;121:491-9.

19. Kazui T, Yamashita K, Washiyama N, Terada H, Bashar AH, Suzuki T, et al Usefulness of antegrade selective cerebral perfusion during aortic arch operations. Ann Thorac Surg. 2002;74:S1806-9; discussion S1825-32.

20. Gutsche JT, Feinman J, Silvay G, Patel PP, Ghadimi K, Landoni G, et al. Practice variations in the conduct of hypothermic circulatory arrest for adult aortic arch repair: focus on an emerging European paradigm. Heart Lung Vessel. 2014;6: 43-51.

21. Urbanski PP, Lenos A, Bougioukakis P, Neophytou I, Zacher M, Diegeler A. Mild-to-moderate hypothermia in aortic arch surgery using circulatory arrest: a change of paradigm? Eur J Cardiothorac Surg. 2012;41:185-91.

22. Hu Z, Wang Z, Ren Z, Wu H, Zhang M, Zhang H, et al. Similar cerebral protective effectiveness of antegrade and retrograde cerebral perfusion combined with deep hypothermia circulatory arrest in aortic arch surgery: a meta-analysis and systematic review of 5060 patients. J Thorac Cardiovasc Surg. 2014;148:544-60.

23. Wilde JT. Hematological consequences of profound hypothermic circulatory arrest and aortic dissection. J Card Surg. 1997;12(2 Suppl):201-6.

24. Leshnower BG, Myung RJ, Kilgo PD, Vassiliades TA, Vega JD, Thourani VH, et al. Moderate hypothermia and unilateral selective antegrade cerebral perfusion: a contemporary cerebral protection strategy for aortic arch surgery. Ann Thorac Surg. 2010;90:547-54.

25. Zierer A, Detho F, Dzemali O, Aybek T, Moritz A, Bakhtiary F. Antegrade cerebral perfusion with mild hypothermia for aortic arch replacement: singlecenter experience in 245 consecutive patients. Ann Thorac Surg. 2011;91: 1868-73.

26. Pacini D, Pantaleo A, Di Marco L, Leone A, Barberio G, Murana G, et al. Visceral organ protection in aortic arch surgery: safety of moderate hypothermia. Eur J Cardiothorac Surg. 2014;46:438-43.

Key Words: warmer temperatures within moderate hypothermia, antegrade cerebral perfusion, arch surgery, elective proximal arch surgery, patient outcomes 